\title{
NASAL RHINOSPORIODIOSIS FROM UTTAR PRADESH (INDIA). A NON-ENDEMIC ZONE: FIRST CASE REPORT
}

\author{
1*Shalini Malhotra, ${ }^{1}$ Om Prakash Bobade, ${ }^{1}$ Ankit Chauhan, ${ }^{2}$ Nripen Vishnoi, ${ }^{1}$ Charoo Hans
}

${ }^{1}$ Department of Microbiology, Dr. Ram Manohar Lohia Hospital and Post Graduate Institute of Medical Research, New Delhi, India; ${ }^{2}$ Department of ENT, Dr. Ram Manohar Lohia Hospital and Post Graduate Institute of Medical Research, New Delhi, India.

Submitted: March 28, 2010; Returned to authors for corrections: April 21, 2010; Approved: November 04, 2010.

\begin{abstract}
Rhinosporiodiosis is a cosmopolitan disease of man and animals, endemic in India and Sri Lanka with main focus of infection in Southern Tamil Nadu. Uttar Pradesh (UP) is not known to be an endemic zone for this disease. We present here the first case of nasal Rhinosporiodiosis from this non-endemic zone.
\end{abstract}

Key words: Rhinosporiodiosis, polypoidal, Uttar Pradesh

Rhinosporiodiosis is a chronic granulomatous infection caused by Rhinosporidium seeberi. This organism had first been regarded as a sporozoan by Malbran, its discoverer in 1892 and as a protozoan later and then as phycomycete by Ashworth in 1923. However through molecular biological analysis of the organism's ribosomal DNA, it was classified into a new clade Mesomycetozoa (1), which includes fish and amphibian pathogens. This was also confirmed using $18 \mathrm{~s} \mathrm{r}$ RNA analysis to finally resolve the debate on taxonomy of this organism particularly that it is not a classic fungus but rather the first known human pathogen from DRIPs clade (Dermocystidium, the rosette agent, Ichthyophonus and Psorospermium) a novel clade of aquatic protistan parasite (1).

The infection is usually acquired by contact with stagnant fresh water such as swimming pools, lakes etc (6). The commonest presentation is in the form of sessile or pedunculated vascular polyps in nose $(70 \%)$ or nasopharynx (5). The disease is endemic in India and Sri lanka with hyper- endemicity in southern districts of Tamil Nadu (4). We present here the first case of nasal rhinosporiodiosis from the resident of Uttar Pradesh which is a non- endemic zone for this disease.

A 30 yrs old male patient resident of Uttar Pradesh presented to the ENT OPD of Dr. Ram Manohar Lohia Hospital and PGIMER Institute New Delhi with complaints of nasal obstruction and mass in the right nasal cavity for the past 6 years .The mass was initially small and slowly it increased in size with gradual progression to nasal obstruction and discomfort .Patient also complained of frequent episodes of nasal bleeding for the past six months. There was no history of any purulent nasal discharge. Patient gave history of bathing in the small stream located nearby his village. There was no history of travel to South India or abroad. There was no family history of the same clinical condition. There was no past history of TB, DM, asthma or allergy. No other significant clinical history could be elicited. On examination, the general physical examination was normal with no obvious external

\footnotetext{
*Corresponding Author. Mailing address: Department of Microbiology, Dr. Ram Manohar Lohia Hospital and Post Graduate Institute of Medical Research, New Delhi, India.; E-mail: drshalinimalhotra@yahoo.com
} 
deformity. On local examination a polypoid friable reddish mass (approx.5cm in size) with irregular surface was visualized on the floor of right nasal cavity attached to lateral wall at the level of posterior end of inferior turbinates. Posterior rhinoscopy revealed irregular reddish mass filling the nasopharynx and extending up to oropharynx. No associated regional lymphadenopathy was observed.CT scan paranasal sinus (PNS) (Figure 1) revealed soft tissue lesion of $5.8 \times 2.3$ cms extending from the naso-lacrimal duct opening along the right inferior turbinate extending up to the posterior nasopharynx. Laboratory investigation revealed hemoglobin (Hb) 15.8 gm\%, Total Leukocyte Count (TLC) 10700/cmm, Differential Leukocyte Count (DLC) $\mathrm{P}_{52}, \mathrm{~L}_{44}, \mathrm{E}_{3}, \mathrm{M}_{1}$ and ESR $4 \mathrm{~mm}$ at the end of one hour. Blood sugar was $91 \mathrm{mg} \%$ and rest of biochemical parameters were within normal limits, Montoux test was negative.

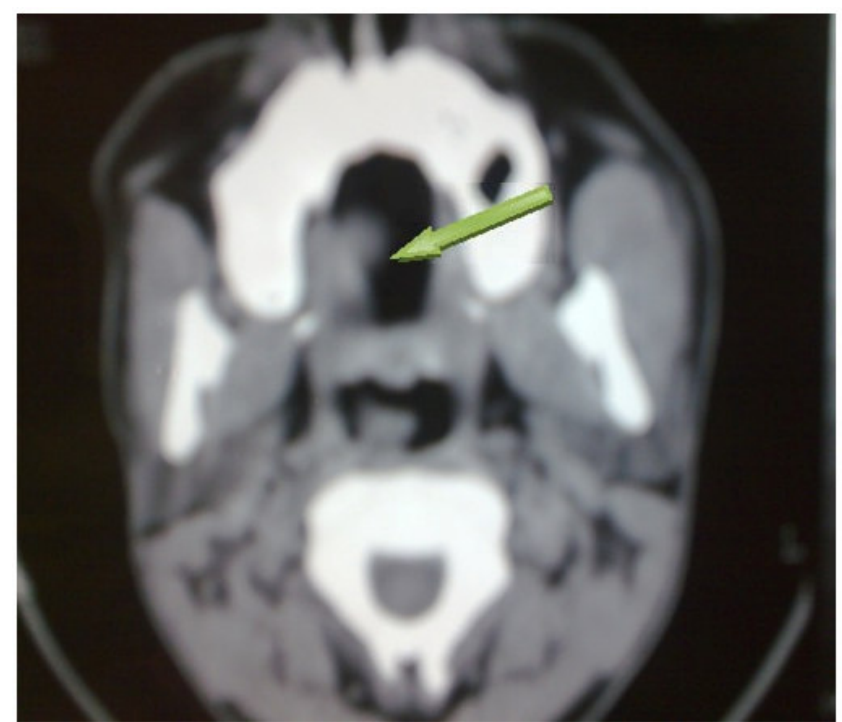

Figure 1. CT scan PNS -showing soft tissue mass along the right inferior turbinate

Based on the above findings a provisional clinical diagnosis of nasal polyp was made and the patient was operated which included endoscopic excision of the nasal mass along with wide cauterization of the polypoidal mucosa of inferior turbinate of the right side of the nose. The nasal mass was sent to microbiology department in saline for fungal and bacterial culture. On gross examination, the mass was pink soft and approximately $5 \times 2 \mathrm{~cm}$ in size. The surface was irregular with yellowish white spots all over its surface. The sample was processed for bacterial and fungal culture as per standard techniques (3). Bacterial culture revealed nonpathogenic bacterial flora of the nose and under $10 \% \mathrm{KOH}$ (Potassium hydroxide) mount, numerous diffusely dispersed sporangiospores $(8-10 \mu)$ were visualized along with intact thick walled large sporangia $(200 \mu)$ characteristically suggestive of Rhinosporidium seeberi (Figure 2). Thus a diagnosis of nasal Rhinosporiodiosis was made and the patient was asked to report to ENT OPD every 6 months for follow-up.

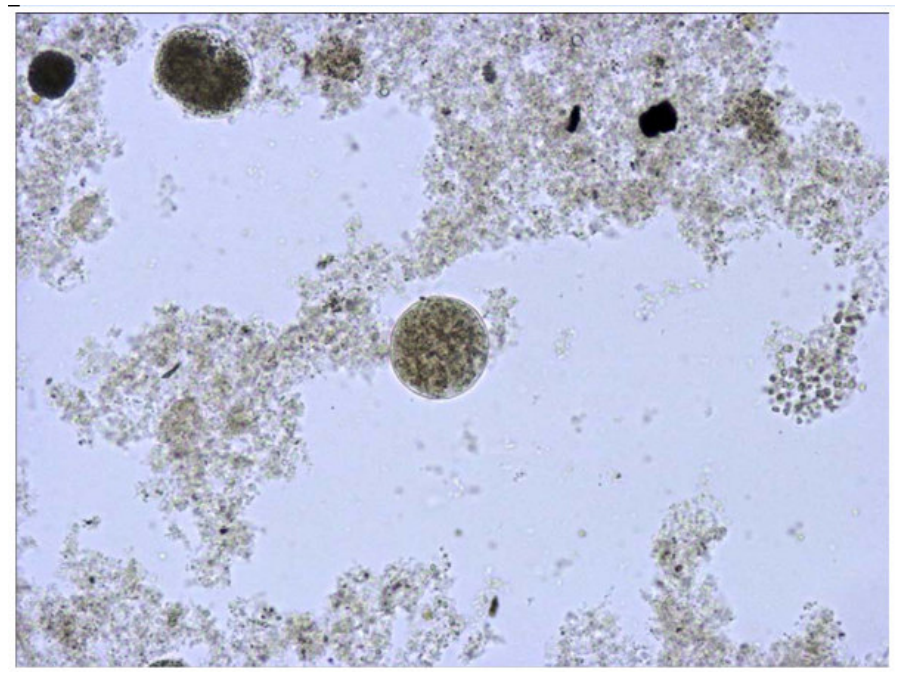

Figure 2. $\mathrm{KOH}$ mount of nasal mass under100x showing numerous sporangia containing endospores.

Rhinosporiodiosis has been known for more than a hundred years since its first description in Argentina. It is an infective chronic granulomatous disease caused by Rhinosporidium seeberi, which affects mainly the mucosa of the nasal cavity pharynx and conjunctiva. India and Sri-lanka 
are two known endemic zones for Rhinosporiodiosis .The disease has also been recognized in other parts of the world like South America \& Egypt. Our case was a resident of UttarPradesh which is not known as endemic zone for this infection .Patient must have acquired the infection while taking bath in the river which is revealed by the history and also which has been implicated as the source of infection in previous studies (2). Endospores contained in water must have penetrated mucosa of the nasal cavity of our patient and matured into sporangium within the sub-mucosa. Rhinosporidium seeberi is difficult to culture in vitro, and hence the diagnosis is established by biopsy of the polyp. We performed the direct microscopy of the specimen under $\mathrm{KOH}$ mount and visualized typical morphology required to make diagnosis (5). For now, the main treatment option is surgical excision of the growth. This however, is associated with recurrence, especially if not completely excised or if autoinoculation has occurred in a satellite site before excision .Hence a wide surgical excision with electro-cautery was advocated in our case due to strong clinical suspicion to reduce the risk of recurrence (1). Thus we conclude that both clinician and laboratory personnel should keep in mind a differential diagnosis of Rhinosporiodiosis while managing the case of nasal mass even if the patient is not from an endemic zone. It is essential to keep a follow up of such patients since the recurrences of the lesion are very common in these cases (5). This will also keep a check on establishment of these infections in naïve non-endemic zone.

\section{REFERENCES}

1. Arseculeratne, S.N. (2002). Recent Advances in Rhinosporiodiosis and Rhinosporidium seeberi. Indian J Med Microbiol. 2(3), 119-131.

2. Balachandran, C.; Muthiah, V.; Moses, J.S. (1990). Incidence and Clinico-pathological studies on Rhinosporiodiosis in Tamil Nadu. $J$ Indian Med. Asso.88, 274.

3. Forbes, B.A.; Sahm, D.F.; Weissfeld, A.S. (1998).Laboratory methods in Basic Mycology. In: Bailey and Scott's Diagnostic Microbiology. Mosby publication. 12th edn, p.871-891.

4. Kindo, A.J.; Sundaram, S. (2007). Unusual presentation of Rhinosporiodiosis - A Case Report. Sri Ramchandra Journal of Medicine. 1(2), 60- 61.

5. Lasser, A.; Smith, H.W. (1976). Rhinosporiodiosis.Arch Otolaryngol. 102, 308-310.

6. Manjunath, M.; Shenoy, B.; Girisha, S.(2007). Cutaneous Rhinosporiodiosis. Indian J Dermatology Venereol Leprol. 73 (3),179181 . 